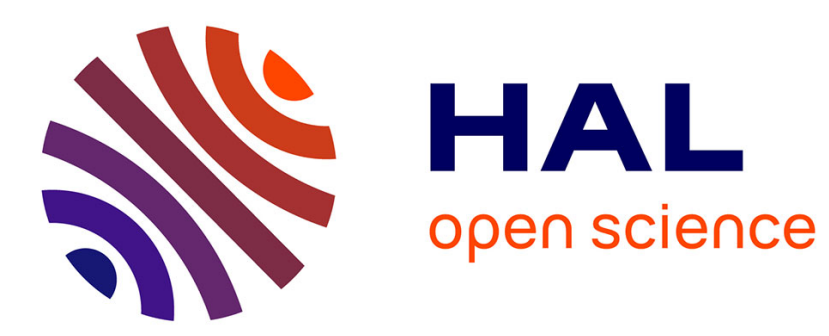

\title{
Gravity waves, cold pockets and CO2 clouds in the Martian mesosphere
}

\author{
Aymeric Spiga, F. Gonzalez-Galindo, M. -A. Lopez-Valverde, F. Forget
}

\section{To cite this version:}

Aymeric Spiga, F. Gonzalez-Galindo, M. -A. Lopez-Valverde, F. Forget. Gravity waves, cold pockets and CO2 clouds in the Martian mesosphere. Geophysical Research Letters, 2012, 39 (2), pp.L02201. 10.1029/2011GL050343. hal-01113118

\section{HAL Id: hal-01113118 https://hal.science/hal-01113118}

Submitted on 4 Feb 2015

HAL is a multi-disciplinary open access archive for the deposit and dissemination of scientific research documents, whether they are published or not. The documents may come from teaching and research institutions in France or abroad, or from public or private research centers.
L'archive ouverte pluridisciplinaire $\mathbf{H A L}$, est destinée au dépôt et à la diffusion de documents scientifiques de niveau recherche, publiés ou non, émanant des établissements d'enseignement et de recherche français ou étrangers, des laboratoires publics ou privés. 


\title{
Gravity waves, cold pockets and $\mathrm{CO}_{2}$ clouds in the Martian mesosphere
}

\author{
A. Spiga, ${ }^{1}$ F. González-Galindo, ${ }^{2}$ M.-Á. López-Valverde, ${ }^{2}$ and F. Forget ${ }^{1}$ \\ Received 15 November 2011; revised 5 December 2011; accepted 12 December 2011; published 20 January 2012.
}

[1] Many independent measurements have shown that extremely cold temperatures are found in the Martian mesosphere. These mesospheric "cold pockets" may result from the propagation of atmospheric waves. Recent observational achievements also hint at such cold pockets by revealing mesospheric clouds formed through the condensation of $\mathrm{CO}_{2}$, the major component of the Martian atmosphere. Thus far, modeling studies addressing the presence of cold pockets in the Martian mesosphere have explored the influence of large-scale circulations. Mesoscale phenomena, such as gravity waves, have received less attention. Here we show through multiscale meteorological modeling that mesoscale gravity waves could play a key role in the formation of mesospheric cold pockets propitious to $\mathrm{CO}_{2}$ condensation. Citation: Spiga, A., F. González-Galindo, M.-Á. López-Valverde, and F. Forget (2012), Gravity waves, cold pockets and $\mathrm{CO}_{2}$ clouds in the Martian mesosphere, Geophys. Res. Lett., 39, L02201, doi:10.1029/2011GL050343.

\section{Introduction and Background}

[2] When topography, convection, fronts, or jet-streams perturb the stratified atmospheric fluid, the buoyancy restoring force gives rise to gravity waves (GWs) which impact both regional and global climate [Alexander et al., 2010]. Despite the omnipresence of GWs in the Martian atmosphere [Briggs and Leovy, 1974], their study is only at the first stages.

[3] Mars GWs have been directly identified through radio occultation [Hinson et al., 1999; Creasey et al., 2006] and accelerometry [Seiff and Kirk, 1976; Keating et al., 1998; Magalhaes et al., 1999; Fritts et al., 2006]. Indirect evidence has been provided by altimeter artefacts caused by $\mathrm{GW}$ clouds [Pettengill and Ford, 2000; Tobie et al., 2003], pressure perturbations [Spiga et al., 2007] and mixed layers induced by GW breaking [Heavens et al., 2010]. All observations indicate that GWs are especially prominent in the mesosphere at $z>60 \mathrm{~km}$, causing temperature and density oscillations over $10 \%$, with vertical/horizontal wavelengths $\left(\lambda_{z}, \lambda_{H}\right) \lesssim$ $(10,200) \mathrm{km}$ [Magalhaes et al., 1999; Fritts et al., 2006].

[4] GWs are thought to be one of the main cause for the presence in the Pathfinder entry profile of extremely cold mesospheric temperatures propitious to local $\mathrm{CO}_{2}$ condensation (hereinafter referred to as "cold pockets") [Schofield et al., 1997]. Following the Pathfinder measurements,

\footnotetext{
${ }^{1}$ Laboratoire de Météorologie Dynamique, Université Pierre et Marie Curie, Institut Pierre-Simon Laplace, Paris, France.

${ }^{2}$ Instituto de Astrofísica de Andalucía, Consejo Superior de Investigaciones Científica, Granada, Spain.

Copyright 2012 by the American Geophysical Union. 0094-8276/12/2011GL050343
}

Clancy and Sandor [1998] detected mesospheric detached hazes and speculated that " $\mathrm{CO}_{2}$ ice clouds should form within the temperature minima of tidal and GWs in the Mars mesosphere".

[5] The presence of daytime $\mathrm{CO}_{2}$ clouds at altitudes $60<z<90 \mathrm{~km}$ has been recently confirmed by Mars Express [Montmessin et al., 2006, 2007; Määttänen et al., 2010], Mars Global Surveyor [Clancy et al., 2007], Mars Odyssey [McConnochie et al., 2010] and Mars Reconnaissance Orbiter [Vincendon et al., 2011]. Those measurements yield a wealth of details on the composition, morphology, variability of mesospheric $\mathrm{CO}_{2}$ clouds. This allows to further constrain the spatial and temporal distribution of mesospheric cold pockets, which are a necessary (not sufficient) condition for the presence of $\mathrm{CO}_{2}$ clouds [González-Galindo et al., 2011].

[6] Here we combine those recent observational achievements with novel modeling diagnostics. Our goal is to revisit the Clancy and Sandor [1998] hypothesis through examining the link between mesospheric GW activity and the occurrence of cold pockets. In section 2, we employ mesoscale modeling to quantify GW-induced mesospheric temperature perturbations. In section 3, we investigate the variability of global meteorological conditions known to impact GW propagation and compare these to the observed variability of mesospheric cold pockets, as inferred from the distribution of $\mathrm{CO}_{2}$ clouds.

\section{Modeling Mesospheric GW Perturbations}

[7] Clancy and Sandor [1998] considered both thermal tides and GWs as plausible phenomena to account for mesospheric cold pockets. González-Galindo et al. [2011] recently concluded through global climate modeling (GCM) that thermal tides yield a particularly cold mesosphere where and when $\mathrm{CO}_{2}$ clouds are observed, yet not cold enough for $\mathrm{CO}_{2}$ condensation to occur. In this section we examine if GWs, unresolved through GCM, could cause mesospheric cold pockets more propitious to $\mathrm{CO}_{2}$ condensation.

[8] To that purpose, we employ the three-dimensional mesoscale meteorological model of Spiga and Forget [2009], whose spatial and temporal resolutions are suitable for GWs. The model features complete Martian physical parameterizations, including an improved treatment for non-local thermodynamic equilibrium (NLTE) radiative processes in the mesosphere/thermosphere [González-Galindo et al., 2009] based on the full model of López-Valverde et al. [2000].

[9] The resulting model has the ability to simulate Martian GWs propagating from lower troposphere to upper mesosphere. The initial temperature profile relies on GCM predictions for springtime afternoons in Meridiani which feature both tidal minima and observed $\mathrm{CO}_{2}$ clouds between 

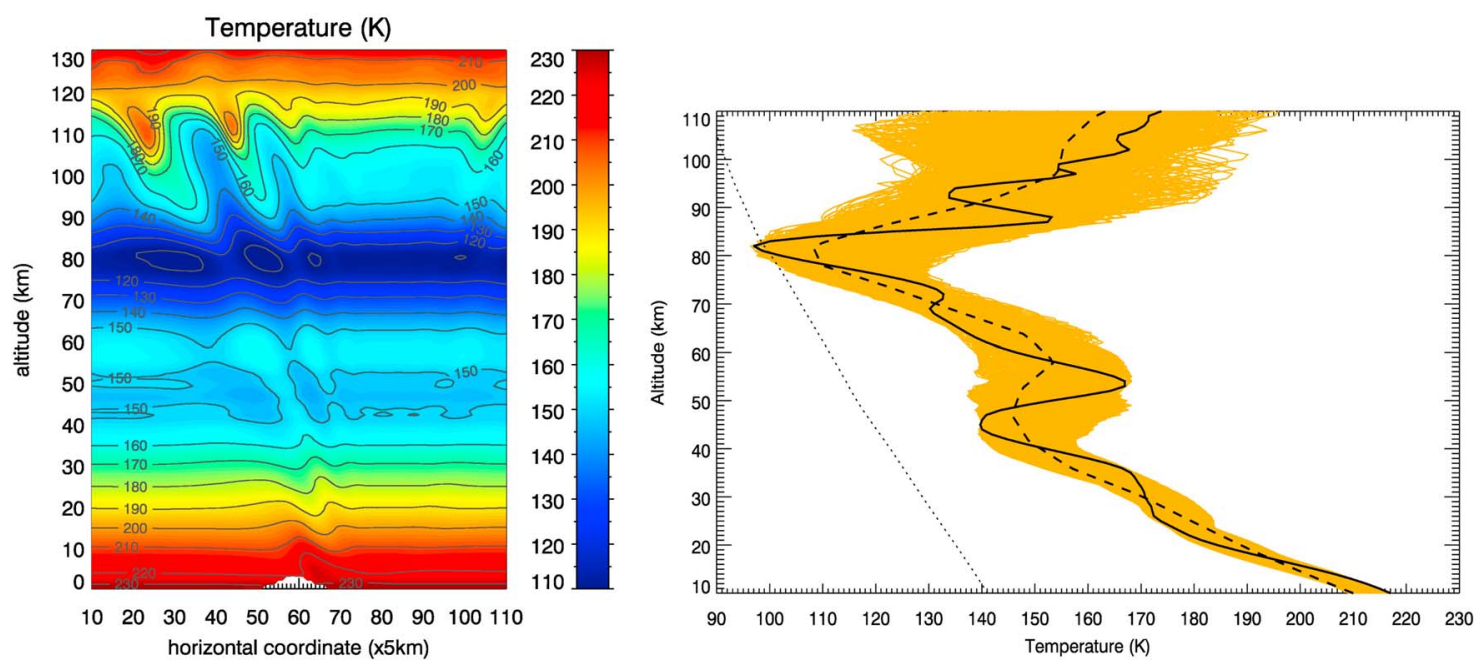

Figure 1. GW packets emitted in the Martian troposphere yield mesospheric temperature perturbations which cause temperature to decrease below the $\mathrm{CO}_{2}$ condensation level. This figure shows results from idealized mesoscale simulations with mountain height $4 \mathrm{~km}$, uniform background wind $30 \mathrm{~m} \mathrm{~s}^{-1}$, dust opacity 0.3 . Initial temperature profile corresponds to GCM predictions at $L_{s}=0^{\circ}-30^{\circ}$, latitude $0^{\circ}$, longitude $0^{\circ}$ and local time 16:00 [González-Galindo et al., 2011, Figure 5]. In the left plot, an $x z$ slice in the domain center shows atmospheric temperature predicted by mesoscale simulations after 1 simulated hour (background wind is from left to right). In the right plot, vertical profiles of temperature in the vicinity of the domain center are shown: dashed line represents initial GCM profile, solid line represents predicted mesoscale profile after 2 simulated hours, orange "envelope" represents a larger set of predicted profiles ranging from 1 to 3 simulated hours and $20 \times 20$ grid points around domain center. $\mathrm{CO}_{2}$ condensation profile $T_{c}$ is superimposed according to Washburn [1948].

$70<z<90 \mathrm{~km}$ [González-Galindo et al., 2011, Figure 5]. Typical GW packets are obtained through considering an uniform background wind impinging a gaussian-shaped mountain. Simulation domain encloses $181 \times 181$ horizontal grid points with $5 \mathrm{~km}$ spacing and 181 vertical levels with $1 \mathrm{~km}$ spacing up to $z=180 \mathrm{~km}$. Diffusive damping is activated for $z>130 \mathrm{~km}$ in a so-called "sponge layer" to prevent spurious GW reflection close to model top [Skamarock and Klemp, 2008]; a $50 \mathrm{~km}$-thick layer is necessary given large GW perturbations in a low density environment. $\mathrm{CO}_{2}$ condensation scheme is deactivated to allow for cold pockets below $\mathrm{CO}_{2}$ condensation temperature. Despite its idealized nature, our GW model is an improvement to existing models, which use either imposed spectra [Eckermann et al., 2011], ad hoc 1D GWs [Parish et al., 2009], or low model top [Rafkin et al., 2001; Tobie et al., 2003].

[10] Results from a typical simulation after a few hours of integration are shown in Figure 1. GW-induced alternating patterns of positive/negative perturbations of temperature $T^{\prime}$ reach $\pm 12 \mathrm{~K}$ in the lower mesosphere. Around $80 \mathrm{~km}$ altitude, these GW perturbations cause mesospheric temperature to fall $2-3 \mathrm{~K}$ below $\mathrm{CO}_{2}$ condensation temperature (Figure 1, right). It is only in combination with large-scale tides that mesoscale GW perturbations yield subcondensation cold pockets in the mesosphere. Subcondensation excursions predicted in Figure 1 are actually likely to be underestimated, because mesospheric GCM temperatures are systematically 5-10 K warmer than SPICAM profiles at this season/location [Forget et al., 2009]. Subcondensation temperature occurs for this particular case at $z \sim 70-80 \mathrm{~km}$, where most $\mathrm{CO}_{2}$ clouds have been observed, and over a depth $\sim 3 \mathrm{~km}$, i.e. the order-of-magnitude for cloud vertical extent in the work of Montmessin et al. [2007]. Higher in the mesosphere $(z>90 \mathrm{~km}), \mathrm{GW}$ perturbations reach $15-20 \%$, in fair agreement with observations.
[11] Although these conclusions appear robust for various simulation settings, GW-induced cold pockets obtained through simulations with lower mountain height or slower incoming wind than in Figure 1 do not fall below the $\mathrm{CO}_{2}$ condensation level. This shows that putative GW perturbations possibly leading to the formation of $\mathrm{CO}_{2}$ clouds are probably above average, though not exceptional. This is consistent with the relative scarcity of observed $\mathrm{CO}_{2}$ clouds. Notwithstanding this, GW temperature perturbations could actually be larger if radiative relaxation processes, or damping rates, were not as efficient in the mesosphere as current NLTE modeling suggests [López-Valverde et al., 2000; Eckermann et al., 2011]. Still our simulations predict mesospheric GW perturbations large enough to yield cold pockets propitious to $\mathrm{CO}_{2}$ condensation.

\section{Variability of Mesospheric GW Activity}

[12] $\mathrm{CO}_{2}$ clouds must be found where and when atmospheric background conditions are favorable to the upward propagation, and subsequent amplification, of GWs emitted in the troposphere as in Figure 1. Apart from dissipative processes (e.g. radiative damping), GW activity is significant in the mesosphere only if GW breaking does not occur in the troposphere or lower mesosphere. The latter happens either through instabilities or critical level filtering, i.e. GW phase speed $c$ becomes comparable to background wind $\langle u\rangle$ and GWs are no longer able to propagate [Lindzen, 1981; Barnes, 1990]. Key background parameters controlling mesoscale GW propagation are thus large-scale horizontal wind $\langle u\rangle$ and static stability $N^{2}=\frac{g}{\langle T\rangle}\left[\frac{d\langle T\rangle}{d z}+\frac{g}{c_{p}}\right]$ where $\langle T\rangle$ is large-scale temperature, $g$ is acceleration of gravity and $c_{p}$ specific heat capacity. 


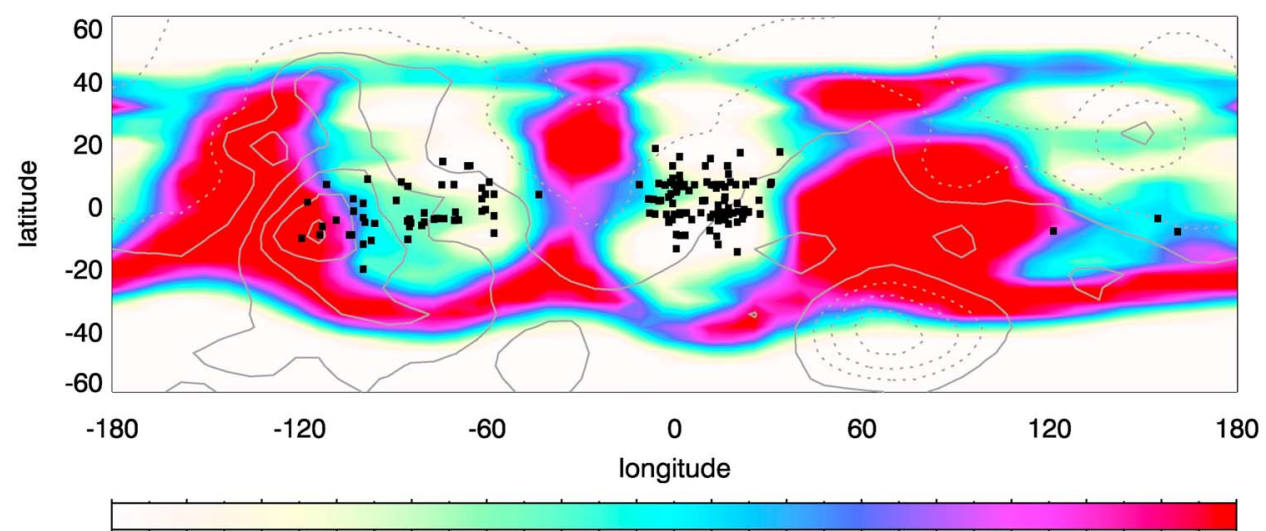

$\begin{array}{llllllllllllllll}-2.0 & -1.9 & -1.8 & -1.7 & -1.6 & -1.5 & -1.4 & -1.3 & -1.2 & -1.1 & -1.0 & -0.9 & -0.8 & -0.7 & -0.6 & -0.5\end{array}$

Figure 2. Mesospheric $\mathrm{CO}_{2}$ clouds are found in regions where $\mathrm{GW}$ emitted in the troposphere can propagate to the mesosphere. This figure displays the map of the maximum saturation index $\mathcal{S}$ reached between the top of the boundary layer $\left(z=10 \mathrm{~km}\right.$ above the local surface) and the highest mesospheric altitude where daytime $\mathrm{CO}_{2}$ clouds have been observed $\left(z=90 \mathrm{~km}\right.$ above zero datum reference). The plotted quantity is $\log _{10}(\mathcal{S})$. Pink to red areas correspond to regions where $\mathcal{S}$ is close enough to 1, i.e. mesospheric GW activity is less likely. White to blue areas correspond to areas where $\mathcal{S}$ is at least one order of magnitude smaller than 1,i.e. significant mesospheric GW activity is more likely. The displayed season is early spring $\left(L_{s}=30^{\circ}\right)$ and local time $L T=15: 00$, when numerous daytime mesospheric $\mathrm{CO}_{2}$ clouds have been detected. The atmospheric fields used to compute $\mathcal{S}$ are extracted from the Mars Climate Database at a resolution of $5^{\circ}$ longitude, $3^{\circ}$ latitude and $\sim 3 \mathrm{~km}$ altitude (http://web.lmd.jussieu.fr/forget/dvd/docs). Mesospheric $\mathrm{CO}_{2}$ clouds observed at altitudes $55<z<90 \mathrm{~km}$ for $L_{s} \pm \delta L_{s}$ with $\delta L_{s}=10^{\circ}$ and $L T \pm \delta L T$ with $\delta L T=01: 00$ are superimposed as black squares. Similar figures at other seasons and local times can be found in the auxiliary material.

[13] Significant mesospheric GW activity is expected at locations, local times and seasons with high $|\langle u\rangle-c|$ and low $N$ in the troposphere and lower mesosphere. Following Hauchecorne et al. [1987], this can be formulated to first order through a "saturation index" $\mathcal{S}$

$$
\mathcal{S}=\frac{T^{\prime}}{T_{m}^{\prime}}=\sqrt{\frac{\alpha N}{\langle\rho\rangle|\langle u\rangle-c|^{3}}} \quad \text { with } \quad \alpha=\frac{F_{0} \lambda_{H}}{2 \pi}
$$

with $\langle\rho\rangle$ the background density, $T^{\prime}\left(T^{\prime}{ }_{m}\right)$ the (maximum) GW perturbation before wave breaking and $F_{0}=\rho\left\langle u^{\prime} w^{\prime}\right\rangle$ the GW vertical momentum flux conserved for non-dissipating GWs (Eliassen-Palm theorem). The formula applies to GWs with midrange frequency $\omega$, for which compressibility and Coriolis effects are negligible. The closest $\mathcal{S}$ is to 1 (or greater values), the more likely upward-propagating GWs from tropospheric sources are to break through criticality or saturation. Conversely, $\mathcal{S}$ at least one order of magnitude smaller than 1 in the troposphere/lower mesosphere is a necessary condition for significant mesospheric GW activity.

[14] To assess the spatial and temporal variability of $\mathcal{S}$, four-dimensional large-scale meteorological fields $N,\langle u\rangle$ and $\rho$ are extracted from the Mars Climate Database (MCD) built upon global-climate modeling with nominal dust scenario (http://web.lmd.jussieu.fr/forget/dvd/docs). A GW packet of $\lambda_{H}=150 \mathrm{~km}$ with $F_{0}=7.5 \times 10^{-7} \mathrm{~J} \mathrm{~m}^{-3}$ is assumed as typical, following Parish et al. [2009], and used to calculate $\alpha$. For simplicity, GW phase speed is set to $c=0 \mathrm{~m} \mathrm{~s}^{-1}$, since most GWs are close enough to stationarity. Alternative values of $\left(\lambda_{H}, F_{0}, c\right)$ yield slightly different values of $\mathcal{S}$, yet similar regional and seasonal contrasts $(\mathcal{S}$ is a qualitative, order-of-magnitude, index rather than an absolute quantitative ratio for wave saturation).

[15] In Figure 2, a global map of the maximum saturation index $\mathcal{S}$ reached in the troposphere/lower mesosphere is shown for early springtime afternoons with observed mesospheric $\mathrm{CO}_{2}$ clouds superimposed. Regions where most mesospheric $\mathrm{CO}_{2}$ clouds are observed at this season, notably two "clusters" in Terra Meridiani and Tharsis/Valles Marineris, correspond to areas where $\mathcal{S}$ is low from troposphere to lower mesosphere, i.e. where upward-propagating GWs emitted in the lower atmosphere are much less likely to become unstable and break before reaching the mesosphere. This is also true for the more isolated cloud events in Aeolis (longitude $120^{\circ} \mathrm{E}$ ). A minority of mesospheric $\mathrm{CO}_{2}$ clouds are observed in areas where saturation index $\mathcal{S}$ is predicted to be high (longitude $-120^{\circ} \mathrm{E}$ ). Those few exceptions are considered as acceptable, within our criterion, given the proximity of those clouds to areas with low $\mathcal{S}$ and the MCD spatial resolution and uncertainties (http://web.lmd.jussieu. fr/forget/dvd/docs).

[16] A closer inspection of MCD vertical profiles for various regions in Figure 2 shows that an inversion of horizontal wind $\langle u\rangle$ (and subsequent decrease towards $c$ ) plays a more prominent role than the variations of atmospheric stability $N$ in yielding large values of saturation $\mathcal{S}$. Conversely, low values for $\mathcal{S}$ are found where strong jets develop in the whole troposphere and lower mesosphere without changing sign, in accordance with studies of GW activity on Earth and Mars [e.g., Barnes, 1990; Leblanc et al., 1995; Heavens et al., 2010].

[17] Figures similar to Figure 2 for other seasons featuring mesospheric $\mathrm{CO}_{2}$ clouds are reported in the auxiliary material: earlier spring, mid-to-late summer, mid-winter, morning clouds in mid-spring. ${ }^{1}$ All cases show that mesospheric $\mathrm{CO}_{2}$ clouds correspond to areas/seasons where/when

${ }^{1}$ Auxiliary materials are available in the HTML. doi:10.1029/ 2011 GL050343. 
the GW saturation index $\mathcal{S}$ is low from troposphere to lower mesosphere. This suggests that upward-propagating GWs, and the induced mesospheric cold pockets, are probably one of the important factors conducive to the formation of mesospheric $\mathrm{CO}_{2}$ clouds.

[18] Mesospheric $\mathrm{CO}_{2}$ clouds do not appear whenever and wherever saturation index $\mathcal{S}$ is predicted to be low. This is expected regardless of observational coverage issues. Firstly, the microphysical processes at play to form $\mathrm{CO}_{2}$ cloud particles in mesospheric cold pockets remain to be explored. Secondly, low $\mathcal{S}$ is not a sufficient condition for GW activity which is also obviously controlled by GW sources. Putative GW activity monitored through radiooccultations at $z<30 \mathrm{~km}$ indicates active tropospheric GW sources within $\pm 25^{\circ}$ latitudes [Creasey et al., 2006, Figure 4], which could account for the latitudinal confinement of $\mathrm{CO}_{2}$ clouds in Figure 2. Thirdly, as is the case in Figure 1, mesospheric GW activity is more likely to yield cold pockets propitious to the formation of $\mathrm{CO}_{2}$ clouds where and when mesospheric temperature are decreased through large-scale meteorological phenomena such as thermal tides. GCM modeling by González-Galindo et al. [2011] suggests that latitudinal, longitudinal and seasonal variability of observed $\mathrm{CO}_{2}$ clouds correlates well with mesospheric temperature minima caused by both nonmigrating and migrating thermal tides. This could explain not only the equatorial confinement of $\mathrm{CO}_{2}$ clouds in northern spring in Figure 2, but also most of the latitudinal variability in Figure S1, which could not be accounted for by $\mathcal{S}$ maps only. Mesospheric $\mathrm{CO}_{2}$ clouds appear to be preferentially found where and when subcondensation cold pockets form under the combined influence (or "constructive interference") of large-scale thermal tides and mesoscale GWs.

\section{Conclusion}

[19] Our conclusions are summarized as follows.

[20] 1. In combination with large-scale dynamical phenomena, mesoscale GWs are crucial for creating mesospheric cold pockets in which $\mathrm{CO}_{2}$ clouds can form.

[21] 2. Most of regions/seasons featuring mesospheric $\mathrm{CO}_{2}$ clouds are characterized by atmospheric conditions favorable to GW upward propagation from tropospheric sources.

[22] A key question to address in future work is how $\mathrm{CO}_{2}$ ice cloud particles form within mesospheric cold pockets. This would involve coupling mesoscale modeling with stateof-the-art microphysical parameterizations. To understand how the major atmospheric component on Mars could condense in high-altitude clouds is likely to broaden the knowledge of both Martian climate and cloud formation processes.

[23] Acknowledgments. We thank N. G. Heavens and A. Medvedev for thorough reviews which improved the manuscript. We are indebted to A. Määttänen, R. T. Clancy, and M. Vincendon for providing data sets and advice about mesospheric $\mathrm{CO}_{2}$ clouds. We also acknowledge S. R. Rafkin, F. Montmessin, and P. Withers for useful discussions about GWs. First steps of this study took place during a scientific visit to Instituto de Astrofísica de Andalucía funded by Junta de Andalucía. A.S. also acknowledges support by IPSL and ESA/CNES.

[24] The Editor thanks Nicholas Heavens and Alexander Medvedev.

\section{References}

Alexander, M. J., et al. (2010), Recent developments in gravity-wave effects in climate models and the global distribution of gravity-wave momentum flux from observations and models, Q. J. R. Meteorol. Soc., 136(650), 1103-1124, doi:10.1002/qj.637.

Barnes, J. R. (1990), Possible effect of breaking gravity waves on the circulation of the middle atmosphere of Mars, J. Geophys. Res., 95, 1401-1421.

Briggs, G. A., and C. B. Leovy (1974), Mariner 9 observations of the Mars north polar hood, Bull. Am. Meteorol. Soc., 55, 278-296.

Clancy, R. T., and B. J. Sandor (1998), $\mathrm{Co}_{2}$ ice clouds in the upper atmosphere of Mars, Geophys. Res. Lett., 25, 489-492.

Clancy, R. T., M. J. Wolff, B. A. Whitney, B. A. Cantor, and M. D. Smith (2007), Mars equatorial mesospheric clouds: Global occurrence and physical properties from Mars Global Surveyor Thermal Emission Spectrometer and Mars Orbiter Camera limb observations, J. Geophys. Res., 112, E04004, doi:10.1029/2006JE002805.

Creasey, J. E., J. M. Forbes, and D. P. Hinson (2006), Global and seasonal distribution of gravity wave activity in Mars' lower atmosphere derived from MGS radio occultation data, Geophys. Res. Lett., 33, L01803, doi:10.1029/2005GL024037.

Eckermann, S. D., J. Ma, and X. Zhu (2011), Scale-dependent infrared radiative damping rates on Mars and their role in the deposition of gravitywave momentum flux, Icarus, 211, 429-442, doi:10.1016/j.icarus. 2010.10.029

Forget, F., F. Montmessin, J.-L. Bertaux, F. González-Galindo, S. Lebonnois, E. Quémerais, A. Reberac, E. Dimarellis, and M. A. López-Valverde (2009), Density and temperatures of the upper Martian atmosphere measured by stellar occultations with Mars Express SPICAM, J. Geophys. Res., 114, E01004, doi:10.1029/2008JE003086.

Fritts, D. C., L. Wang, and R. H. Tolson (2006), Mean and gravity wave structures and variability in the Mars upper atmosphere inferred from Mars Global Surveyor and Mars Odyssey aerobraking densities, J. Geophys. Res., 111, A12304, doi:10.1029/2006JA011897.

González-Galindo, F., F. Forget, M. A. López-Valverde, M. Angelats i Coll, and E. Millour (2009), A ground-to-exosphere Martian general circulation model: 1 . Seasonal, diurnal and solar cycle variation of thermospheric temperatures, J. Geophys. Res., 114, E04001, doi:10.1029/ 2008JE003246.

González-Galindo, F., A. Määttänen, F. Forget, and A. Spiga (2011), The Martian mesosphere as revealed by $\mathrm{CO} 2$ clouds observations and general circulation modeling, Icarus, 216, 10-22.

Hauchecorne, A., M. L. Chanin, and R. Wilson (1987), Mesospheric temperature inversion and gravity wave breaking, Geophys. Res. Lett., 14, 933-936, doi:10.1029/GL014i009p00933.

Heavens, N. G., et al. (2010), Convective instability in the Martian middle atmosphere, Icarus, 208, 574-589, doi:10.1016/j.icarus.2010.03.023.

Hinson, D. P., M. Flasar, R. A. Simpson, J. D. Twicken, and G. L. Tyler (1999), Initial results from radio occultation measurements with Mars global surveyor, J. Geophys. Res., 104, 26,997-27,012.

Keating, G. M., et al. (1998), The structure of the upper atmosphere of Mars: In situ accelerometer measurements from Mars Global Surveyor, Science, 279, 1672-1676.

Leblanc, T., A. Hauchecorne, M.-L. Chanin, C. Rodgers, F. Taylor, and N. Livesey (1995), Mesospheric temperature inversions as seen by ISAMS in December 1991, Geophys. Res. Lett., 22, 1485-1488, doi:10.1029/94GL03274.

Lindzen, R. S. (1981), Turbulence and stress owing to gravity wave and tidal breakdown, J. Geophys. Res., 86, 9707-9714.

López-Valverde, M. A., R. M. Haberle, and M. López-Puertas (2000), Non-LTE radiative mesospheric study for Mars Pathfinder entry, Icarus, 146, 360-365, doi:10.1006/icar.2000.6388.

Määttänen, A., et al. (2010), Mapping the mesospheric $\mathrm{CO}_{2}$ clouds on Mars: MEx/OMEGA and MEx/HRSC observations and challenges for atmospheric models, Icarus, 209, 452-469, doi:10.1016/j.icarus.2010.05.017.

Magalhaes, J. A., J. T. Schofield, and A. Seiff (1999), Results of the Mars Pathfinder atmospheric structure investigation, J. Geophys. Res., 104, 8943-8956, doi:10.1029/1998JE900041.

McConnochie, T. H., J. F. Bell, D. Savransky, M. J. Wolff, A. D. Toigo, H. Wang, M. I. Richardson, and P. R. Christensen (2010), THEMIS-VIS observations of clouds in the Martian mesosphere: Altitudes, wind speeds, and decameter-scale morphology, Icarus, 210, 545-565, doi:10.1016/j. icarus.2010.07.021

Montmessin, F., et al. (2006), Subvisible $\mathrm{CO}_{2}$ ice clouds detected in the mesosphere of Mars, Icarus, 183, 403-410, doi:10.1016/j.icarus.2006.03.015.

Montmessin, F., B. Gondet, J.-P. Bibring, Y. Langevin, P. Drossart, F. Forget, and T. Fouchet (2007), Hyperspectral imaging of convective $\mathrm{CO}_{2}$ ice clouds in the equatorial mesosphere of Mars, J. Geophys. Res., 112, E11S90, doi:10.1029/2007JE002944.

Parish, H. F., G. Schubert, M. P. Hickey, and R. L. Walterscheid (2009), Propagation of tropospheric gravity waves into the upper atmosphere of Mars, Icarus, 203, 28-37, doi:10.1016/j.icarus.2009.04.031.

Pettengill, G. H., and P. G. Ford (2000), Winter clouds over the North Martian Polar Cap, Geophys. Res. Lett., 27, 609-613. 
Rafkin, S. C. R., R. M. Haberle, and T. I. Michaels (2001), The Mars Regional Atmospheric Modeling System: Model description and selected simulations, Icarus, 151, 228-256.

Schofield, J. T., D. Crisp, J. R. Barnes, R. M. Haberle, J. A. Magalhaães, J. R. Murphy, A. Seiff, S. Larsen, and G. Wilson (1997), The Mars Pathfinder Atmospheric Structure Investigation/Meteorology (ASI/MET) experiment, Science, 278, 1752-1757.

Seiff, A., and D. B. Kirk (1976), Structure of the Mars' atmosphere up to 100 kilometers from the entry measurements of Viking 2, Science, 194, $1300-1303$.

Skamarock, W. C., and J. B. Klemp (2008), A time-split nonhydrostatic atmospheric model for weather research and forecasting applications, J. Comput. Phys., 227, 3465-3485, doi:10.1016/j.jcp.2007.01.037.

Spiga, A., and F. Forget (2009), A new model to simulate the Martian mesoscale and microscale atmospheric circulation: Validation and first results, J. Geophys. Res., 114, E02009, doi:10.1029/2008JE003242.

Spiga, A., et al. (2007), Remote sensing of surface pressure on Mars with the Mars Express/OMEGA spectrometer: 2. Meteorological maps, J. Geophys. Res., 112, E08S16, doi:10.1029/2006JE002870.
Tobie, G., F. Forget, and F. Lott (2003), Numerical simulation of the winter polar wave clouds observed by Mars Global Surveyor Mars Orbiter Laser Altimeter, Icarus, 164, 33-49.

Vincendon, M., C. Pilorget, B. Gondet, S. Murchie, and J. Bibring (2011), Observations of mesospheric $\mathrm{CO}_{2}$ and $\mathrm{H}_{2} \mathrm{O}$ clouds on Mars, J. Geophys. Res., 116, E00J02, doi:doi:10.1029/2011JE003827.

Washburn, E. (1948), International Critical Tables of Numerical Data, Physics, Chemistry, and Technology, vol. 3, McGraw Hill, New York.

F. Forget and A. Spiga, Laboratoire de Météorologie Dynamique, Université Pierre et Marie Curie, Institut Pierre-Simon Laplace, F-75252 Paris, France.

F. González-Galindo and M.-Á. López-Valverde, Instituto de Astrofísica de Andalucía, Consejo Superior de Investigaciones Científica, Camino Bajo de Hutor, 24, ES-18080 Granada, Spain. 